\title{
Schultz and Modified Schultz Polynomials of Cog-Complete Bipartite Graphs
}

\author{
Ahmed Mohammed Ali, Haitham Nashwan Mohammed \\ Department of Mathematics, College of Computer Sciences and Mathematics, University of Mosul, Mosul, Iraq \\ Email address: \\ ahmedmucscm79@gmail.com (A. M. Ali), haytham.nashwan@yahoo.com (H. N. Mohammed) \\ To cite this article: \\ Ahmed Mohammed Ali, Haitham Nashwan Mohammed. Schultz and Modified Schultz Polynomials of Cog-Complete Bipartite Graphs. \\ Applied and Computational Mathematics. Vol. 6, No. 6, 2017, pp. 259-264. doi: 10.11648/j.acm.20170606.14
}

Received: September 9, 2017; Accepted: November 9, 2017; Published: December 18, 2017

\begin{abstract}
Let $\mathrm{G}$ be a simple connected graph, the vertex- set and edge- set of $\mathrm{G}$ are denoted by $V(G)$ and $E(G)$, respectively. The molecular graph $\mathrm{G}$, the vertices represent atoms and the edges represent bonds. In graph theory, we have many invariant polynomials and many invariant indices of a connected graph G. Topological indices based on the distance between the vertices of a connected graph are widely used in theoretical chemistry to establish relation between the structure and the properties of molecules. The coefficients of polynomials are also important in the knowledge some properties in application chemistry. The Schultz and modified Schultz polynomials, Schultz and modified Schultz indices and average distance of Schultz and modified Schultz of Cog-complete bipartite graphs are obtained in this paper.
\end{abstract}

Keywords: Schultz and Modified Schultz Polynomials, Cog-Complete Bipartite Graphs, Topological Indices, Boundary Average Distance

\section{Introduction}

Suppose that $G=(V(G), E(G))$ is a simple undirected connected graph of order $p=p(G)$ and size $q=q(G)$. A graph $\mathrm{G}$ is called an n-partite graph, $n \geq 2$, if it possible to partition vertex- set $V(G)$ in to non empty subsets $V_{1}, V_{2}, \ldots, V_{n}$ (called partite sets) such that every element of $E(G)$ joins a vertex of $\mathrm{V}_{\mathrm{r}}$ to a vertex of $\mathrm{V}_{\mathrm{t}}$, for all $\mathrm{r} \neq \mathrm{t}$ for $\mathrm{n}=2$, such graphs are called bipartite graphs. A complete bipartite graph $\mathrm{G}$ is a 2- partite graph with petite sets $V_{1}$ and $\mathrm{V}_{2}$ having the added property that if $u \in V_{1}$ and $v \in V_{2}$, then $u v \in V(G)$ with two partite sets $\mathrm{V}_{1}$ and $\mathrm{V}_{2}$ denoted by $\mathrm{K}_{\mathrm{m}, \mathrm{n}}$ (or $\mathrm{K}(\mathrm{m}, \mathrm{n})$, where $\left|\mathrm{V}_{1}\right|=\mathrm{m}$ and $\left|\mathrm{V}_{2}\right|=\mathrm{n}$. The distance between any two vertices $u$ and $v$ of $G$ is the length of a shortest (u,v)-path in a connected graph $\mathrm{G}$, denoted by $d(u, v)$. In particular, if $u=v$, then $d(u, v)=0$. Topological indices in biology and chemistry was used for the first time in 1947 when chemist Harold Wiener [1] introduced Wiener index to demonstrate correlations between physicochemical properties of organic compounds of molecular graphs. The Wiener index is represent the total distance of a connected graph $G$, i.e. $W(G)=\sum_{u, v \in V(G)} d(u, v)$, in which the summation is taken over all unordered pairs $\{u, v\}$ of distinct vertices of $\mathrm{G}$. The diameter of $\mathrm{G}$ is the greatest distance in $\mathrm{G}$ and will be denoted by $\delta$. The number of pairs of vertices of $G$ that are distance $\mathrm{k}$ is denoted by $d(G, K)$. Distance is an important concept in graph theory and it has applications to computer science, chemistry, and a variety of other fields [2-6].

For the definitions of concepts and notations used in this papers, vertices with distance $\mathrm{k}$ such that $\left|D_{k}(G)\right|=d(G, k)$ and $\sum_{k=1}^{\delta} d(G, k)=\left(\begin{array}{l}p \\ 2\end{array}\right)$, where $\left(\begin{array}{l}p \\ 2\end{array}\right)$ is representation the number of unordered pairs of distinct vertices in $\mathrm{G}$.

In chemical graph theory, there are two important polynomials are distinguished. Introduced:

The Schultz polynomial of a graph $\mathrm{G}$ is defined as:

$$
\operatorname{Sc}(G ; x)=\sum_{u, v \in V(G)}\left(\delta_{u}+\delta_{v}\right) x^{d(u, v)},
$$

and modified Schultz polynomial of a graph $\mathrm{G}$ is defined as:

$$
S c^{*}(G ; x)=\sum_{u, v \in V(G)}\left(\delta_{u} \delta_{v}\right) x^{d(u, v)} .
$$

These based structure descriptors and their polynomials were extensively studied and computed before [7-9].

The molecular topological index (Schultz index) was introduced by Harry P. Schultz in 1989 [10] and the modified Schultz index was defined by S. Klavžar and I. Gutman in 1997 [11]. 
The Schultz index is defined as:

$$
\operatorname{Sc}(G)=\sum_{u, v \in V(G)}\left(\delta_{u}+\delta_{v}\right) d(u, v),
$$

and modified Schulttz index is defined as:

$$
S c^{*}(G)=\sum_{u, v \in V(G)}\left(\delta_{u} \delta_{v}\right) d(u, v) .
$$

where the summation for all above are taken over all unordered pairs of distinct vertices in $\mathrm{V}(\mathrm{G})$.

The indices of Schultz and modified Schultz can be obtained by the derivative of Schultz and modified Schultz polynomials with respect to $\mathrm{x}$ at $\mathrm{x}=1$, respectively, i.e.:

$$
S c(G)=\left.\frac{d}{d x} S c(G ; x)\right|_{x=1} \text {, and } S c^{*}(G)=\left.\frac{d}{d x} S c^{*}(G ; x)\right|_{x=1} \text {. }
$$

The average distance of a connected graph $G$ with respect Schultz and modified Schultz are defined as:

$$
\overline{S c(G)}=S c(G) /\left(\begin{array}{l}
p \\
2
\end{array}\right) \text {, and } \overline{S c^{*}(G)}=S c^{*}(G) /\left(\begin{array}{l}
p \\
2
\end{array}\right) .
$$

In 2005, Gutman find many relations between Hosoya, Schultz and modified Schultz polynomials of a tree graph and some properties [12]. Bo Zhou [13], find some lower and upper bounds for the Schultz index of a graph G.

The Schultz and modified Schultz polynomial of some special graphs are summarized in the following theorem (See [14]). In addition, we have found the Schultz and modified Schultz polynomials of some Cog- special graphs which it under publication.

In 2013, Hassani et al. computed the Schultz polynomials of isomeres of $\mathrm{C}_{100}$ Fullerene by GAP program [15].

The Schultz indices have been shown to be a useful molecular descriptors in the design of molecules with desired properties, reader can be see the papers [16-18].
Finally, Farahani found Schultz and modified Schultz polynomials and topological indices of benzene molecules PAHs, coronene polycyclic aromatic hydro carbons and some Haray graphs $[8,19,20]$ in 2017, Guo and Zhow studies the propeties of degree distance and Gutman index of uniform hypergraphs [21].

\section{Main Results}

Definition: A Cog- complete bipartite graph $K_{m, n}^{c}$ is the graphconstructed frombipartite complete graph $K_{m, n}, m, n \geq 2$, of vertices set $\left\{\mathrm{u}_{1}, \mathrm{u}_{2}, \ldots, \mathrm{u}_{\mathrm{m}}, \mathrm{v}_{1}, \mathrm{v}_{2}, \ldots, \mathrm{v}_{\mathrm{n}}\right\}$ with $m+n-2$ of additional vertices $\left\{w_{1}, w_{2}, \ldots, w_{m-1}, y_{1}, y_{2}, \ldots, y_{n-1}\right\}$, and $2 \mathrm{~m}+2 \mathrm{n}-4$ of additional edges $\left\{w_{i} u_{i}, w_{i} u_{i+1}: i=1,2, \ldots, m-\right.$ $1\} \cup\left\{y_{i} v_{i}, y_{i} v_{i+1}: i=1,2, \ldots, n-1\right\}$, see Figure 1 .

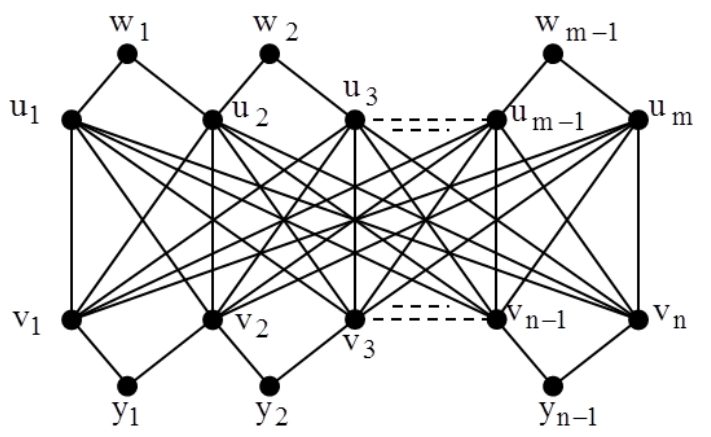

Figure 1. A Cog-complete bipartite graph $K_{m, n}^{c}$.

From Clearly that $p\left(K_{m, n}^{c}\right)=2 m+2 n-2, q\left(K_{m, n}^{c}\right)=$ $m(n+2)+2(n-2)$ and $\delta=\operatorname{diam} K_{m, n}^{c}=4, \quad$ for all $m, n \geq 4$.

Theorem 2.1: For all $m, n \geq 4$, then

$$
\begin{array}{r}
S c\left(K_{m, n}^{c} ; x\right)=\{m n(m+n+8)+4(m+n-5)\} x+2\left\{m^{2}(n+1)+(n-4)(n+1)+m(n+3)(n-1)\right\} x^{2}+ \\
\left\{m^{2}(n+4)+m\left(n^{2}-2 n-16\right)+4\left(n^{2}-4 n+7\right)\right\} x^{3}+2\{m(m-5)+n(n-5)+12\} x^{4} .(1) \\
S c^{*}\left(K_{m, n}^{c} ; x\right)=\{m n(m n+2 m+2 n+8)-20\} x+\{m n(m n+(7 m / 2)+(7 n / 2)-4)+2 m(m-4)+2 n(n-4)+ \\
2\} x^{2}+2\left\{m^{2}(n+2)+n^{2}(m+2)-2(m n+4 m+4 n-9)\right\} x^{3}+2\{m(m-5)+n(n-5)+12\} x^{4} .(2)
\end{array}
$$

Proof: For all vertices $z_{1} z_{2} \in V\left(K_{m, n}^{c}\right)$, there is $d\left(z_{1}, z_{2}\right)=k, k=1,2,3,4$.

From clearly that $\sum_{i=1}^{4}\left|D_{i}\right|=(2 m+2 n-3)(m+n-1)$.

We will have four partitions for proof:

P1. If $d\left(z_{1}, z_{2}\right)=1$, then $\left|\mathrm{D}_{1}\right|=\mathrm{m}(\mathrm{n}+2)+2(\mathrm{n}-2)$ and is equal to $q\left(K_{m, n}^{c}\right)$, we have ten subsets of it:

$$
\begin{aligned}
& \text { P1.1. }\left|\left\{\left(\mathrm{u}_{1(\mathrm{~m})}, \mathrm{w}_{1(\mathrm{~m}-1)}\right): \delta_{\mathrm{u}_{1}(\mathrm{~m})}+\delta_{\mathrm{w}_{1}(\mathrm{~m}-1)}=\mathrm{n}+3 \& \delta_{\mathrm{u}_{1}(\mathrm{~m})} \delta_{\mathrm{w}_{1}(\mathrm{~m}-1)}=2(\mathrm{n}+1)\right\}\right|=2 . \\
& \text { P1.2. }\left|\left\{\left(\mathrm{w}_{\mathrm{i}}, \mathrm{u}_{\mathrm{i}+1}\right): \delta_{\mathrm{w}_{\mathrm{i}}}+\delta_{\mathrm{u}_{\mathrm{i}+1}}=\mathrm{n}+4 \& \delta_{\mathrm{w}_{\mathrm{i}}} \delta_{\mathrm{u}_{\mathrm{i}+1}}=2(\mathrm{n}+2), 1 \leq \mathrm{i} \leq \mathrm{m}-2\right\}\right|=\mathrm{m}-2 . \\
& \text { P1.3. }\left|\left\{\left(\mathrm{w}_{\mathrm{i}}, \mathrm{u}_{\mathrm{i}}\right): \delta_{\mathrm{w}_{\mathrm{i}}}+\delta_{\mathrm{u}_{\mathrm{i}}}=\mathrm{n}+4 \& \delta_{\mathrm{w}_{\mathrm{i}}} \delta_{\mathrm{u}_{\mathrm{i}}}=2(\mathrm{n}+2), 2 \leq \mathrm{i} \leq \mathrm{m}-1\right\}\right|=\mathrm{m}-2 . \\
& \text { P1.4. }\left|\left\{\left(\mathrm{v}_{1(\mathrm{n})}, \mathrm{y}_{1(\mathrm{n}-1)}\right): \delta_{\mathrm{v}_{1}(\mathrm{n})}+\delta_{\mathrm{y}_{1}(\mathrm{n}-1)}=\mathrm{m}+3 \& \delta_{\mathrm{v}_{1}(\mathrm{n})} \delta_{\mathrm{y}_{1}(\mathrm{n}-1)}=2(\mathrm{~m}+1)\right\}\right|=2 . \\
& \text { P1.5. }\left|\left\{\left(\mathrm{y}_{\mathrm{i}}, \mathrm{v}_{\mathrm{i}+1}\right): \delta_{\mathrm{y}_{\mathrm{i}}}+\delta_{\mathrm{v}_{\mathrm{i}+1}}=\mathrm{m}+4 \& \delta_{\mathrm{y}_{\mathrm{i}}} \delta_{\mathrm{v}_{\mathrm{i}+1}}=2(\mathrm{~m}+2), 1 \leq \mathrm{i} \leq \mathrm{n}-2\right\}\right|=\mathrm{n}-2 . \\
& \text { P1.6. }\left|\left\{\left(\mathrm{y}_{\mathrm{i}}, \mathrm{v}_{\mathrm{i}}\right): \delta_{\mathrm{y}_{\mathrm{i}}}+\delta_{\mathrm{v}_{\mathrm{i}}}=\mathrm{m}+4 \& \delta_{\mathrm{y}_{\mathrm{i}}} \delta_{\mathrm{v}_{\mathrm{i}}}=2(\mathrm{~m}+2), 2 \leq \mathrm{i} \leq \mathrm{n}-1\right\}\right|=\mathrm{n}-2 .
\end{aligned}
$$


P1.7. $\left|\left\{\left(\mathrm{u}_{\mathrm{i}}, \mathrm{v}_{\mathrm{j}}\right): \delta_{\mathrm{u}_{\mathrm{i}}}+\delta_{\mathrm{v}_{\mathrm{j}}}=\mathrm{m}+\mathrm{n}+2 \& \delta_{\mathrm{u}_{\mathrm{i}}} \delta_{\mathrm{v}_{\mathrm{j}}}=(\mathrm{m}+1)(\mathrm{n}+1), \mathrm{i}=1, \mathrm{~m}, \mathrm{j}=1, \mathrm{n}\right\}\right|=4$.

P1.8. $\left|\left\{\left(\mathrm{u}_{\mathrm{i}}, \mathrm{v}_{\mathrm{j}}\right): \delta_{\mathrm{u}_{\mathrm{i}}}+\delta_{\mathrm{v}_{\mathrm{j}}}=\mathrm{m}+\mathrm{n}+3 \& \delta_{\mathrm{u}_{\mathrm{i}}} \delta_{\mathrm{v}_{\mathrm{j}}}=(\mathrm{m}+1)(\mathrm{n}+2), 2 \leq \mathrm{i} \leq \mathrm{m}-1, \mathrm{j}=1, \mathrm{n}\right\}\right|=2(\mathrm{~m}-2)$.

P1.9. $\left|\left\{\left(u_{i}, v_{j}\right): \delta_{u_{i}}+\delta_{v_{j}}=m+n+3 \& \delta_{u_{i}} \delta_{v_{j}}=(m+2)(n+1), i=1, m, 2 \leq j \leq n-1\right\}\right|=2(n-2)$.

P1.10. $\left|\left\{\left(u_{i}, v_{j}\right): \delta_{u_{i}}+\delta_{v_{j}}=m+n+4 \& \delta_{u_{i}} \delta_{v_{j}}=(m+2)(n+2), 2 \leq \mathrm{i} \leq m-1,2 \leq j \leq n-1\right\}\right|=(m-2)(n-2)$. P2. If $d\left(z_{1}, z_{2}\right)=2$, then $\left|D_{2}\right|=(4 m n+m(m-1)+n(n-1)-8) / 2$, we have twelve subsets of it:

P2.1. $\left|\left\{\left(\mathrm{y}_{\mathrm{i}}, \mathrm{y}_{\mathrm{i}+1}\right): \delta_{\mathrm{y}_{\mathrm{i}}}+\delta_{\mathrm{y}_{\mathrm{i}+1}}=4 \& \delta_{\mathrm{y}_{\mathrm{i}}} \delta_{\mathrm{y}_{\mathrm{i}+1}}=4,1 \leq \mathrm{i} \leq \mathrm{n}-2\right\}\right|=\mathrm{n}-2$.

P2.2. $\left|\left\{\left(\mathrm{w}_{\mathrm{i}}, \mathrm{w}_{\mathrm{i}+1}\right): \delta_{\mathrm{w}_{\mathrm{i}}}+\delta_{\mathrm{w}_{\mathrm{i}+1}}=4 \& \delta_{\mathrm{w}_{\mathrm{i}}} \delta_{\mathrm{w}_{\mathrm{i}+1}}=4,1 \leq \mathrm{i} \leq \mathrm{m}-2\right\}\right|=\mathrm{m}-2$.

P2.3. $\left|\left\{\left(\mathrm{v}_{1}, \mathrm{v}_{\mathrm{n}}\right): \delta_{\mathrm{v}_{1}}+\delta_{\mathrm{v}_{\mathrm{n}}}=2(\mathrm{~m}+1) \& \delta_{\mathrm{v}_{1}} \delta_{\mathrm{v}_{\mathrm{n}}}=(\mathrm{m}+1)^{2}\right\}\right|=1$.

P2.4. $\left|\left\{\left(\mathrm{v}_{\mathrm{i}}, \mathrm{v}_{\mathrm{j}}\right): \delta_{\mathrm{v}_{\mathrm{i}}}+\delta_{\mathrm{v}_{\mathrm{j}}}=2 \mathrm{~m}+3 \& \delta_{\mathrm{v}_{\mathrm{i}}} \delta_{\mathrm{v}_{\mathrm{j}}}=(\mathrm{m}+1)(\mathrm{m}+2), \mathrm{i}=1, \mathrm{n}, 2 \leq \mathrm{j} \leq \mathrm{n}-1\right\}\right|=2(\mathrm{n}-2)$.

P2.5. $\left|\left\{\left(\mathrm{v}_{\mathrm{i}}, \mathrm{v}_{\mathrm{j}}\right): \delta_{\mathrm{v}_{\mathrm{i}}}+\delta_{\mathrm{v}_{\mathrm{j}}}=2(\mathrm{~m}+2) \& \delta_{\mathrm{v}_{\mathrm{i}}} \delta_{\mathrm{v}_{\mathrm{j}}}=(\mathrm{m}+2)^{2}, 2 \leq \mathrm{i} \leq \mathrm{n}-2, \mathrm{i}+1 \leq \mathrm{j} \leq \mathrm{n}-1\right\}\right|=(\mathrm{n}-2)(\mathrm{n}-3) / 2$.

P2.6. $\left|\left\{\left(\mathrm{u}_{1}, \mathrm{u}_{\mathrm{m}}\right): \delta_{\mathrm{u}_{1}}+\delta_{\mathrm{u}_{\mathrm{m}}}=2(\mathrm{n}+1) \& \delta_{\mathrm{u}_{1}} \delta_{\mathrm{u}_{\mathrm{m}}}=(\mathrm{n}+1)^{2}\right\}\right|=1$.

P2.7. $\left|\left\{\left(u_{i}, u_{j}\right): \delta_{u_{i}}+\delta_{u_{j}}=2 n+3 \& \delta_{u_{i}} \delta_{u_{j}}=(n+1)(n+2), i=1, m, 2 \leq j \leq m-1\right\}\right|=2(m-2)$.

P2.8. $\left|\left\{\left(\mathrm{u}_{\mathrm{i}}, \mathrm{u}_{\mathrm{j}}\right): \delta_{\mathrm{u}_{\mathrm{i}}}+\delta_{\mathrm{u}_{\mathrm{j}}}=2(\mathrm{n}+2) \& \delta_{\mathrm{u}_{\mathrm{i}}} \delta_{\mathrm{u}_{\mathrm{j}}}=(\mathrm{n}+2)^{2}, 2 \leq \mathrm{i} \leq \mathrm{m}-2, \mathrm{i}+1 \leq \mathrm{j} \leq \mathrm{m}-1\right\}\right|=(\mathrm{m}-2)(\mathrm{m}-3) / 2$.

P2.9. $\left|\left\{\left(\mathrm{v}_{\mathrm{i}}, \mathrm{w}_{\mathrm{j}}\right): \delta_{\mathrm{v}_{\mathrm{i}}}+\delta_{\mathrm{w}_{\mathrm{j}}}=\mathrm{m}+3 \& \delta_{\mathrm{v}_{\mathrm{i}}} \delta_{\mathrm{w}_{\mathrm{j}}}=2(\mathrm{~m}+1), \mathrm{i}=1, \mathrm{n}, 1 \leq \mathrm{j} \leq \mathrm{m}-1\right\}\right|=2(\mathrm{~m}-1)$

P2.10. $\left|\left\{\left(\mathrm{v}_{\mathrm{i}}, \mathrm{w}_{\mathrm{j}}\right): \delta_{\mathrm{v}_{\mathrm{i}}}+\delta_{\mathrm{w}_{\mathrm{j}}}=\mathrm{m}+4 \& \delta_{\mathrm{v}_{\mathrm{i}}} \delta_{\mathrm{w}_{\mathrm{j}}}=2(\mathrm{~m}+2), 2 \leq \mathrm{i} \leq \mathrm{n}-1,1 \leq \mathrm{j} \leq \mathrm{m}-1\right\}\right|=(\mathrm{m}-1)(\mathrm{n}-2)$.

P2.11. $\left|\left\{\left(u_{i}, y_{j}\right): \delta_{u_{i}}+\delta_{y_{j}}=n+3 \& \delta_{u_{i}} \delta_{y_{j}}=2(n+1), i=1, m, 1 \leq j \leq n-1\right\}\right|=2(n-1)$.

P2.12. $\left|\left\{\left(\mathrm{u}_{\mathrm{i}}, \mathrm{y}_{\mathrm{j}}\right): \delta_{\mathrm{u}_{\mathrm{i}}}+\delta_{\mathrm{y}_{\mathrm{j}}}=\mathrm{n}+4 \& \delta_{\mathrm{u}_{\mathrm{i}}} \delta_{\mathrm{y}_{\mathrm{j}}}=2(\mathrm{n}+2), 2 \leq \mathrm{i} \leq \mathrm{m}-1,1 \leq \mathrm{j} \leq \mathrm{n}-1\right\}\right|=(\mathrm{m}-2)(\mathrm{n}-1)$.

P3. If $d\left(z_{1}, z_{2}\right)=3$, then $\left|D_{3}\right|=m n+m(m-4)+n(n-4)+5$, we have seven subsets of it:

P3.1. $\left|\left\{\left(\mathrm{u}_{1}, \mathrm{w}_{\mathrm{j}}\right): \delta_{\mathrm{u}_{1}}+\delta_{\mathrm{w}_{\mathrm{j}}}=\mathrm{n}+3 \& \delta_{\mathrm{u}_{1}} \delta_{\mathrm{w}_{\mathrm{j}}}=2(\mathrm{n}+1), 2 \leq \mathrm{j} \leq \mathrm{m}-1\right\}\right|=\mathrm{m}-2$.

P3.2. $\left|\left\{\left(\mathrm{u}_{\mathrm{m}}, \mathrm{w}_{\mathrm{j}}\right): \delta_{\mathrm{u}_{\mathrm{m}}}+\delta_{\mathrm{w}_{\mathrm{j}}}=\mathrm{n}+3 \& \delta_{\mathrm{u}_{\mathrm{m}}} \delta_{\mathrm{w}_{\mathrm{j}}}=2(\mathrm{n}+1), 1 \leq \mathrm{j} \leq \mathrm{m}-2\right\}\right|=\mathrm{m}-2$.

P3.3. $\left|\left\{\left(\mathrm{u}_{\mathrm{i}}, \mathrm{w}_{\mathrm{j}}\right): \delta_{\mathrm{u}_{\mathrm{i}}}+\delta_{\mathrm{w}_{\mathrm{j}}}=\mathrm{n}+4 \& \delta_{\mathrm{u}_{\mathrm{i}}} \delta_{\mathrm{w}_{\mathrm{j}}}=2(\mathrm{n}+2), 2 \leq \mathrm{i} \leq \mathrm{m}-1,1 \leq \mathrm{j} \leq \mathrm{m}-1,|\mathrm{i}-\mathrm{j}| \neq 0,1\right\}\right|=(\mathrm{m}-2)(\mathrm{m}-3)$.

P3.4. $\left|\left\{\left(\mathrm{v}_{1}, \mathrm{y}_{\mathrm{j}}\right): \delta_{\mathrm{v}_{1}}+\delta_{\mathrm{y}_{\mathrm{j}}}=\mathrm{m}+3 \& \delta_{\mathrm{v}_{1}} \delta_{\mathrm{y}_{\mathrm{j}}}=2(\mathrm{~m}+1), 2 \leq \mathrm{j} \leq \mathrm{n}-1\right\}\right|=\mathrm{n}-2$.

P3.5. $\left|\left\{\left(\mathrm{v}_{\mathrm{n}}, \mathrm{y}_{\mathrm{i}}\right): \delta_{\mathrm{v}_{\mathrm{n}}}+\delta_{\mathrm{y}_{\mathrm{i}}}=\mathrm{m}+3 \& \delta_{\mathrm{v}_{\mathrm{n}}} \delta_{\mathrm{y}_{\mathrm{i}}}=2(\mathrm{~m}+1), 1 \leq \mathrm{i} \leq \mathrm{n}-2\right\}\right|=\mathrm{n}-2$.

P3.6. $\left|\left\{\left(v_{i}, y_{j}\right): \delta_{v_{i}}+\delta_{y_{j}}=m+4 \& \delta_{v_{i}} \delta_{y_{j}}=2(m+2), 2 \leq \mathrm{i} \leq n-1,1 \leq j \leq n-1,|i-j| \neq 0,1\right\}\right|=(n-2)(n-3)$.

P3.7. $\left|\left\{\left(w_{i}, y_{j}\right): \delta_{w_{i}}+\delta_{y_{j}}=4 \& \delta_{w_{i}} \delta_{y_{j}}=4,1 \leq \mathrm{i} \leq \mathrm{m}-1,1 \leq \mathrm{j} \leq \mathrm{n}-1\right\}\right|=(\mathrm{m}-1)(\mathrm{n}-1)$.

P4. If $\left(z_{1}, z_{2}\right)=4$, then $\left|D_{3}\right|=(m(m-5)+n(n-5)+12) / 2$, we have two subsets of it:

P4.1. $\left|\left\{\left(\mathrm{w}_{\mathrm{i}}, \mathrm{w}_{\mathrm{j}}\right): \delta_{\mathrm{w}_{\mathrm{i}}}+\delta_{\mathrm{w}_{\mathrm{j}}}=4 \& \delta_{\mathrm{w}_{\mathrm{i}}} \delta_{\mathrm{w}_{\mathrm{j}}}=4,1 \leq \mathrm{i} \leq \mathrm{m}-3, \mathrm{i}+2 \leq \mathrm{j} \leq \mathrm{m}-1\right\}\right|=(\mathrm{m}-2)(\mathrm{m}-3) / 2$.

P4.2. $\left|\left\{\left(\mathrm{y}_{\mathrm{i}}, \mathrm{y}_{\mathrm{i}+2}\right): \delta_{\mathrm{y}_{\mathrm{i}}}+\delta_{\mathrm{y}_{\mathrm{i}+2}}=4 \& \delta_{\mathrm{y}_{\mathrm{i}}} \delta_{\mathrm{y}_{\mathrm{i}+2}}=4,1 \leq \mathrm{i} \leq \mathrm{n}-3\right\}\right|=(\mathrm{n}-2)(\mathrm{n}-3) / 2$. 
From P1-P4 and after the computational processes we get the two equations (1) and (2).

Corollary 2.2: For all $m, n \geq 4$, then

$$
\begin{gathered}
S c\left(K_{m, n}^{c}\right)=8 m^{2}(n+3)+8 n^{2}(m+3)+10 m n-96(m+n)+144 . \\
S c^{*}\left(K_{m, n}^{c}\right)=3 m n(m n-8)+3 m^{2}(5 n+8)+3 n^{2}(5 m+8)-104(m+n)+188 .
\end{gathered}
$$

Proof:

To get equations (3) and (4), we derivative the equations (1) and (2) with respect to $x$ and then compensation of the value of $x$ by 1 .

Corollary 2.3: For all $m, n \geq 4$, then

1. $\overline{S c\left(K_{m, n}^{c}\right)}=\left(8 m^{2}(n+3)+8 n^{2}(m+3)+10 m n-96(m+n)+144\right) /(m+n-1)(2 m+2 n-3)$.

2. $\overline{S c^{*}\left(K_{m, n}^{c}\right)}=\left(3 m n(m n-8)+3 m^{2}(5 n+8)+3 n^{2}(5 m+8)-104(m+n)+188\right) /(m+n-1)(2 m+2 n-3)$.

Proof: obvious

Corollary 2.4: For all $m=n \geq 4$, then

$$
\begin{gathered}
S c\left(K_{m, n}^{c} ; x\right)=2\left(m^{3}+4 m^{2}+4 m-10\right) x+4\left(m^{3}+2 m^{2}-3 m-2\right) x^{2}+2\left(m^{3}+3 m^{2}-16 m+14\right) x^{3}+4(m-3)(m-2) x^{4} . \\
S c^{*}\left(K_{m, n}^{c} ; x\right)=\left(m^{4}+4 m^{3}+8 m^{2}-20\right) x+\left(m^{4}+7 m^{3}-16 m+2\right) x^{2}+4\left(m^{3}-8 m+9\right) x^{3}+4(m-3)(m-2) x^{4} .
\end{gathered}
$$

Proof: from possible to obtain formulas (5) and (6) from formulas (1) and (2) by equality $n, m(m=n)$ and after simplified processes.

Corollary 2.5: For all $m=n \geq 4$, then

1. $S c\left(K_{m, n}^{c}\right)=16 m^{3}+58 m^{2}-192 m+144$.

2. $S c^{*}\left(K_{m, n}^{c}\right)=3 m^{4}+30 m^{3}+24 m^{2}-208 m+188$.

Proof: similar to proof corollary 2.2 .

Corollary 2.6: For all $m=n \geq 4$, then

1. $14.5<\overline{S c\left(K_{m, n}^{c}\right)}<2 m+10$.

2. $26.7<\overline{S c^{*}\left(K_{m, n}^{c}\right)}<3\left(4 m^{2}+45 m+87\right) / 32$.

When $n=2$ and $n=3$ for all $m \geq 4$, we obtain the same formulas in the Theorem 2.1 and corollary 2.2 after off setting the value $n=2$ and $n=3$ for all $m \geq 4$, in the Theorem 2.1 and corollary 2.2 respectively.

As special case, if $n=2, m \geq 4$, then $K_{m, n}^{c}$ is shown in

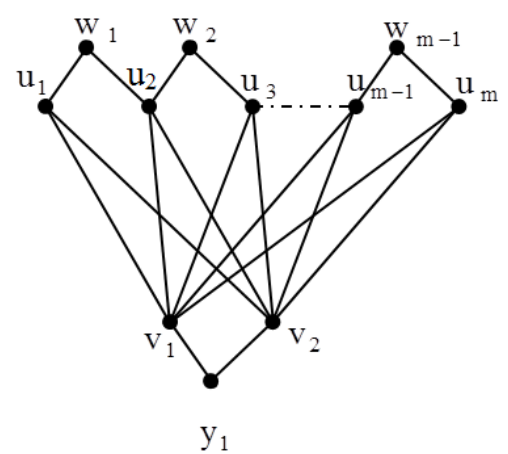

Figure 2. A Cog-complete bipartite graph $K_{m, 2}^{c}$. Figure 2.

$$
\begin{aligned}
& S c\left(K_{m, 2}^{c} ; x\right)=2\left(m^{2}+12 m-6\right) x+2\left(3 m^{2}+5 m-6\right) x^{2}+2\left(3 m^{2}-8 m+6\right) x^{3}+2(m-3)(m-2) x^{4} . \\
& S c^{*}\left(K_{m, 2}^{c} ; x\right)=4\left(2 m^{2}+6 m-5\right) x+\left(13 m^{2}-2 m-6\right) x^{2}+4\left(2 m^{2}-6 m+5\right) x^{3}+2(m-3)(m-2) x^{4} .
\end{aligned}
$$

Proof: For every vertices $z_{1} z_{2} \in V\left(K_{m, 2}^{c}\right)$, there is $\mathrm{d}\left(\mathrm{z}_{1}, \mathrm{z}_{2}\right)=k, k=1,2,3,4$, and obvious $\sum_{i=1}^{4}\left|D_{i}\right|=(2 m+1)(m+1)$.

We will have four partitions for proof:

P1. If $d\left(z_{1}, z_{2}\right)=1$, then $\left|\mathrm{D}_{1}\right|=4 \mathrm{~m}$ and is equal to $q\left(K_{m, 2}^{c}\right)$, we have six subsets of it:

$$
\begin{gathered}
\text { P1.1. }\left|\left\{\left(\mathrm{u}_{1(\mathrm{~m})}, \mathrm{w}_{1(\mathrm{~m}-1)}\right): \delta_{\mathrm{u}_{1}(\mathrm{~m})}+\delta_{\mathrm{w}_{1}(\mathrm{~m}-1)}=5 \& \delta_{\mathrm{u}_{1}(\mathrm{~m})} \delta_{\mathrm{w}_{1}(\mathrm{~m}-1)}=6\right\}\right|=2 . \\
\text { P1.2. }\left|\left\{\left(\mathrm{w}_{\mathrm{i}}, \mathrm{u}_{\mathrm{i}}\right): \delta_{\mathrm{w}_{\mathrm{i}}}+\delta_{\mathrm{u}_{\mathrm{i}}}=6 \& \delta_{\mathrm{w}_{\mathrm{i}}} \delta_{\mathrm{u}_{\mathrm{i}}}=8,2 \leq \mathrm{i} \leq \mathrm{m}-1\right\}\right|=\mathrm{m}-2 . \\
\text { P1.3. }\left|\left\{\left(\mathrm{w}_{\mathrm{i}}, \mathrm{u}_{\mathrm{i}+1}\right): \delta_{\mathrm{w}_{\mathrm{i}}}+\delta_{\mathrm{u}_{\mathrm{i}+1}}=6 \& \delta_{\mathrm{w}_{\mathrm{i}}} \delta_{\mathrm{u}_{\mathrm{i}+1}}=8,1 \leq \mathrm{i} \leq \mathrm{m}-2\right\}\right|=\mathrm{m}-2 . \\
\text { P1.4. }\left|\left\{\left(\mathrm{u}_{\mathrm{i}}, \mathrm{v}_{\mathrm{j}}\right): \delta_{\mathrm{u}_{\mathrm{i}}}+\delta_{\mathrm{v}_{\mathrm{j}}}=\mathrm{m}+4 \& \delta_{\mathrm{u}_{\mathrm{i}}} \delta_{\mathrm{v}_{\mathrm{j}}}=3(\mathrm{~m}+1), \mathrm{i}=1, \mathrm{~m}, \mathrm{j}=1,2\right\}\right|=4 . \\
\text { P1.5. }\left|\left\{\left(\mathrm{u}_{\mathrm{i}}, \mathrm{v}_{\mathrm{j}}\right): \delta_{\mathrm{u}_{\mathrm{i}}}+\delta_{\mathrm{v}_{\mathrm{j}}}=\mathrm{m}+5 \& \delta_{\mathrm{u}_{\mathrm{i}}} \delta_{\mathrm{v}_{\mathrm{j}}}=4(\mathrm{~m}+1), 2 \leq \leq \mathrm{m}-1, \mathrm{j}=1,2\right\}\right|=2(\mathrm{~m}-2) . \\
\text { P1.6. }\left|\left\{\left(\mathrm{y}_{1}, \mathrm{v}_{\mathrm{i}}\right): \delta_{\mathrm{y}_{1}}+\delta_{\mathrm{v}_{\mathrm{i}}}=\mathrm{m}+3 \& \delta_{\mathrm{y}_{1}} \delta_{\mathrm{v}_{\mathrm{i}}}=2(\mathrm{~m}+2), \mathrm{i}=1,2\right\}\right|=2 . \\
\text { P2. If } d\left(z_{1}, z_{2}\right)=2 \text {, then }\left|\mathrm{D}_{2}\right|=\left(\mathrm{m}^{2}+7 \mathrm{~m}-6\right) / 2 \text {, we have eight subsets of it: }
\end{gathered}
$$




$$
\begin{aligned}
& \text { P2.1. }\left|\left\{\left(\mathrm{w}_{\mathrm{i}}, \mathrm{w}_{\mathrm{i}+1}\right): \delta_{\mathrm{w}_{\mathrm{i}}}+\delta_{\mathrm{w}_{\mathrm{i}+1}}=4 \& \delta_{\mathrm{w}_{\mathrm{i}}} \delta_{\mathrm{w}_{\mathrm{i}+1}}=4,1 \leq \mathrm{i} \leq \mathrm{m}-2\right\}\right|=\mathrm{m}-2 . \\
& \text { P2.2. }\left|\left\{\left(\mathrm{v}_{\mathrm{i}}, \mathrm{w}_{\mathrm{j}}\right): \delta_{\mathrm{v}_{\mathrm{i}}}+\delta_{\mathrm{w}_{\mathrm{j}}}=\mathrm{m}+3 \& \delta_{\mathrm{v}_{\mathrm{i}}} \delta_{\mathrm{w}_{\mathrm{j}}}=2(\mathrm{~m}+1), \mathrm{i}=1,2,1 \leq \mathrm{j} \leq \mathrm{m}-1\right\}\right|=2(\mathrm{~m}-1) \text {. } \\
& \text { P2.3. }\left|\left\{\left(\mathrm{u}_{\mathrm{i}}, \mathrm{y}_{1}\right): \delta_{\mathrm{u}_{\mathrm{i}}}+\delta_{\mathrm{y}_{1}}=5 \& \delta_{\mathrm{u}_{\mathrm{i}}} \delta_{\mathrm{y}_{1}}=6, \mathrm{i}=1,2\right\}\right|=2 \text {. } \\
& \text { P2.4. }\left|\left\{\left(\mathrm{u}_{\mathrm{i}}, \mathrm{y}_{1}\right): \delta_{\mathrm{u}_{\mathrm{i}}}+\delta_{\mathrm{y}_{1}}=6 \& \delta_{\mathrm{u}_{\mathrm{i}}} \delta_{\mathrm{y}_{1}}=8,2 \leq \mathrm{i} \leq \mathrm{m}-1\right\}\right|=\mathrm{m}-2 \text {. } \\
& \text { P2.5. }\left|\left\{\left(\mathrm{u}_{1}, \mathrm{u}_{\mathrm{m}}\right): \delta_{\mathrm{u}_{1}}+\delta_{\mathrm{u}_{\mathrm{m}}}=6 \& \delta_{\mathrm{u}_{1}} \delta_{\mathrm{u}_{\mathrm{m}}}=9\right\}\right|=1 \text {. } \\
& \text { P2.6. }\left|\left\{\left(\mathrm{u}_{\mathrm{i}}, \mathrm{u}_{\mathrm{j}}\right): \delta_{\mathrm{u}_{\mathrm{i}}}+\delta_{\mathrm{u}_{\mathrm{j}}}=7 \& \delta_{\mathrm{u}_{\mathrm{i}}} \delta_{\mathrm{u}_{\mathrm{j}}}=12,2 \leq \mathrm{i} \leq \mathrm{m}-1, \mathrm{j}=1, \mathrm{~m}\right\}\right|=2(\mathrm{~m}-2) \text {. } \\
& \text { P2.7. }\left|\left\{\left(\mathrm{u}_{\mathrm{i}}, \mathrm{u}_{\mathrm{j}}\right): \delta_{\mathrm{u}_{\mathrm{i}}}+\delta_{\mathrm{u}_{\mathrm{j}}}=8 \& \delta_{\mathrm{u}_{\mathrm{i}}} \delta_{\mathrm{u}_{\mathrm{j}}}=16,2 \leq \mathrm{i} \leq \mathrm{m}-2, \mathrm{i}+1 \leq \mathrm{j} \leq \mathrm{m}-1\right\}\right|=(\mathrm{m}-2)(\mathrm{m}-3) / 2 \text {. } \\
& \text { P2.8. }\left|\left\{\left(\mathrm{v}_{1}, \mathrm{v}_{2}\right): \delta_{\mathrm{v}_{1}}+\delta_{\mathrm{v}_{2}}=2(\mathrm{~m}+1) \& \delta_{\mathrm{v}_{1}} \delta_{\mathrm{v}_{2}}=(\mathrm{m}+1)^{2}\right\}\right|=1 \text {. } \\
& \text { P3. If } d\left(z_{1}, z_{2}\right)=3 \text {, then }\left|D_{3}\right|=(m-1)^{2} \text {, we have four subsets of it: } \\
& \text { P3.1. }\left|\left\{\left(\mathrm{y}_{1}, \mathrm{w}_{\mathrm{i}}\right): \delta_{\mathrm{y}_{1}}+\delta_{\mathrm{w}_{\mathrm{i}}}=4 \& \delta_{\mathrm{y}_{1}} \delta_{\mathrm{w}_{\mathrm{i}}}=4,1 \leq \mathrm{i} \leq \mathrm{m}-1\right\}\right|=\mathrm{m}-1 \text {. } \\
& \text { P3.2. }\left|\left\{\left(\mathrm{u}_{1}, \mathrm{w}_{\mathrm{i}}\right): \delta_{\mathrm{u}_{1}}+\delta_{\mathrm{w}_{\mathrm{i}}}=5 \& \delta_{\mathrm{u}_{1}} \delta_{\mathrm{w}_{\mathrm{i}}}=6,2 \leq \mathrm{i} \leq \mathrm{m}-1\right\}\right|=\mathrm{m}-2 \text {. } \\
& \text { P3.3. }\left|\left\{\left(\mathrm{u}_{\mathrm{m}}, \mathrm{w}_{\mathrm{i}}\right): \delta_{\mathrm{u}_{\mathrm{m}}}+\delta_{\mathrm{w}_{\mathrm{i}}}=5 \& \delta_{\mathrm{u}_{\mathrm{m}}} \delta_{\mathrm{w}_{\mathrm{i}}}=6,1 \leq \mathrm{i} \leq \mathrm{m}-2\right\}\right|=\mathrm{m}-2 \text {. } \\
& \text { P3.4. }\left|\left\{\left(\mathrm{u}_{\mathrm{i}}, \mathrm{w}_{\mathrm{j}}\right): \delta_{\mathrm{u}_{\mathrm{i}}}+\delta_{\mathrm{w}_{\mathrm{j}}}=6 \& \delta_{\mathrm{u}_{\mathrm{i}}} \delta_{\mathrm{w}_{\mathrm{j}}}=8,2 \leq \mathrm{i} \leq \mathrm{m}-1,1 \leq \mathrm{j} \leq \mathrm{m}-1,|\mathrm{i}-\mathrm{j}| \neq 0,1\right\}\right|=(\mathrm{m}-2)(\mathrm{m}-3) \text {. } \\
& \text { P4. If }\left(z_{1}, z_{2}\right)=4 \text {, then }\left|D_{3}\right|=((m-2)+(m-3)+12) / 2 \text {, we have: } \\
& \left|\left\{\left(\mathrm{w}_{\mathrm{i}}, \mathrm{w}_{\mathrm{j}}\right): \delta_{\mathrm{w}_{\mathrm{i}}}+\delta_{\mathrm{w}_{\mathrm{j}}}=4 \& \delta_{\mathrm{w}_{\mathrm{i}}} \delta_{\mathrm{w}_{\mathrm{j}}}=4,1 \leq \mathrm{i} \leq \mathrm{m}-3, \mathrm{i}+2 \leq \mathrm{j} \leq \mathrm{m}-1\right\}\right|=(\mathrm{m}-2)(\mathrm{m}-3) / 2 \\
& S c\left(K_{3,3}^{c} ; x\right)=130 x+136 x^{2}+40 x^{3} . \\
& \text { 2. } S c^{*}\left(K_{2,2}^{c} ; x\right)=60 x+42 x^{2}+4 x^{3} \text {. } \\
& \operatorname{Sc}^{*}\left(K_{3,2}^{c} ; x\right)=124 x+105 x^{2}+20 x^{3} . \\
& \operatorname{Sc}^{*}\left(K_{3,3}^{c} ; x\right)=241 x+224 x^{2}+48 x^{3} .
\end{aligned}
$$
Theorem 2.1.

Corollary 2.9: For $m \geq 4$, we have:

$1.11 .3<\overline{S c\left(K_{m, 2}^{c}\right)}<20$.

2. $16.9<\overline{S c^{*}\left(K_{m, 2}^{c}\right)}<33$.

Theorem 2.10: For $m \geq 4$, we have:

1. $S c\left(K_{m, 3}^{c} ; x\right)=\left(3 m^{2}+37 m-8\right) x+\left(8 m^{2}+24 m-\right.$ 8) $x^{2}+\left(7 m^{2}-13 m+16\right) x^{3}+\left(2 m^{2}-10 m+12\right) x^{4}$.

2. $S c^{*}\left(K_{m, 3}^{c} ; x\right)=\left(15 m^{2}+42 m-20\right) x+\left\{\left(43 m^{2}+23 m-\right.\right.$ 8) $/ 2\} x^{2}+\left(10 m^{2}-22 m+24\right) x^{3}+\left(2 m^{2}-10 m+12\right) x^{4}$.

Proof: By the same way proof the Theorem 2.1.

Corollary 2.11: For $m \geq 4$, we have:

1. $S c\left(K_{m, 3}^{c}\right)=48 m^{2}+6 m+72$.

2. $S c^{*}\left(K_{m, 3}^{c}\right)=96 m^{2}-41 m+92$.

Corollary 2.12: For $m \geq 4$, we have:

$1.14<\overline{S c\left(K_{m, 3}^{c}\right)}<24$.

2. $25<\overline{S c^{*}\left(K_{m, 3}^{c}\right)}<48$.

Remark:

1. $S c\left(K_{2,2}^{c} ; x\right)=44 x+32 x^{2}+4 x^{3}$.

$$
S c\left(K_{3,2}^{c} ; x\right)=78 x+72 x^{2}+18 x^{3} .
$$

\section{Conclusion}

In this paper we managed to find the Schultz and modified Schultz polynomials and Schultz and modified Schultz indices of Cog- complete bipartite graph $K_{m, n}^{c}$ of order $2 m+2 m-2, m, n \geq 2$. Also, we given the boundary lower and upper of average distance of Schultz and modified Schultz of Cog- complete bipartite graph $K_{m, n}^{c}$.

\section{References}

[1] Wiener, H.; (1947), "Structural Determination of Paraffin Boiling Points" J. Amer. Chem. Soc., 69, 17-20.

[2] Diudea, M. V. (ed.); (2001). QSPR/QSAR Studies by Molecular Descriptors, Nova, Huntington, New York.

[3] Diudea, M. V., Gutman, I. and J"antschi, L.; (2001). Molecular Topology, Nova, Huntington, New York.

[4] Chartrand, G. and Lesniak, L.; (1986). Graphs and Digraphs, 2nd ed., Wadsworth and Brooks/Cole, California. 
[5] Devillers, J. and Balaban, A. T. (eds.); (1999). Topological Indices and Related Descriptors in QSAR and QSPR, Gordon \& Breach, Amsterdam.

[6] Diestel, R.; (2000). Graph Theory, electronic ed., SpringerVerlag, New York.

[7] Farahaini M. R., (2013); "Hosoya, Schultz Modified Schultz Polynomials and their Topological Indices of Benzene Molecules: First Members of polycyclic Aromatic Hydro Carbons (PAHs)"; International Journal of theoretical chemistry Vol. 1, No. 2, pp. 6-9.

[8] Farahaini M. R., On the Schultz Polynomial, Modified Schultz Polynomial, Hosoya polynomial and Wiener Index of Circumcoronene Series of Benzenoid. Journal of Applied Mathematics of infromatics, (2013), 31 (5-6).

[9] Haoer, R. S., Atan, K. A., Khalaf, A. M., Rushdan, M. and Hasni, R (2016); Eccentric Connectivity Index of Some Chemical Trees, International Journal of Pure and Applied Mathematics, Vol. 106, No. 1, pp. 157-170.

[10] Schultz, H. P. (1989), "Topological Organic Chemistry 1". Graph theory and topological indices of alkanes. J. Chem. Inf. Copmut. Sci. 29, 227-228.

[11] Klavžar, S. and Gutman, I. (1997), "Wiener number of vertex -weighted graphs and a chemical application". Disc. Appl. Math. 80, 73-81.

[12] Gutman, I. (2005), "Some Relations Between Distance- Based Polynomials of Trees". Bull. A cod. Serbe. Sci. Arts 131, pp. $1-7$.

[13] Bo Zhou, (2006), "Bounds for the Schultz Molecular Topol- ogical Index" MATCH Commun. Math Comput. Chem. Vol. 56, pp. 189-194.

[14] Behmaram, A, Yousefi- Azari, H. and Ashrafi, A. R., (2011); "Some New Results on Distance-Based Polynomials", MATH. Commun. Math. Comput. Chem. 65, 39-50.

[15] Hassani, G., Iranmanesh, A. and Mirzaie, S. (2013), "Schultz and Modified Schultz Polynomials of $\mathrm{C}_{100}$ Fullerene". MATCH Commun. Math. Comput. Chem. Vol. 69, pp. 87-92.

[16] Iranmanesh, A. and Ali zadeh, Y., (2009), "Computing Szeged and Schultz Indices of HAC3C7C9[p,q] Nanotube by Gap program. Digest Biostructures, 4, pp. 67-72.

[17] Heydar. A., (2010), "Schultz Index of Regular Dendrimers" optoelectronics and Advanced Materials: Rapid Communications, 4, pp. 2209-2211.

[18] Heydari, A., (2010), “On the Modified Schultz Index of C4C8(S) Nanotubes and Nanotours. Digest Journal of Nanomatrial and Biostructures, 5, pp. 51-56.

[19] Farahaini M. R., (2014); "Schultz and Modified Schultz Polyn- omials of Coronene Polycyclic Aromatic Hydro carbons", International Letters of chemistry, Physics and Astronomy, Vol. 32, pp. 1-10.

[20] Farahani M. R., (2013); "On the Schultz and Modified Schultz Polynomials of Some Harary Graphs", International Journal of Applications of Discrete Mathematics; Vol. 1, No. 1, pp. 0108 .

[21] Guo. H. and Zhow B.; (2017), "Properties of Degree Distance and Gutman Index of Uniform Hypergraphs" MATCH Commun. Math. Comput Chem. 78, pp. 213-220. 